\title{
A Cognitive-Evolutionary Model for the Evolution of Teaching
}

\author{
Rafael Ventura ${ }^{1}$ and Erol Akçay ${ }^{2}$ \\ ${ }^{1}$ Social and Cultural Evolution Working Group, University of \\ Pennsylvania \\ ${ }^{2}$ Department of Biology, University of Pennsylvania
}

\begin{abstract}
Mechanisms for social learning have rightly been the focus of much work in cultural evolution. But mechanisms for teaching-mechanisms that determine what information is available for learners to learn in the first place - are equally important to cultural evolution, especially in the case of humans. Here, we propose a simple model of teaching in the context of skill transmission. Our model derives the evolutionary cost and benefit of teaching by explicitly representing cognitive aspects of skill transmission. Results show that there is an "explain-exploit" trade-off inherent to teaching, which can give rise to an opportunity cost that goes beyond any direct cost that it may also entail. We then discuss how this opportunity cost can cause teaching mechanisms to be self-limiting, suggesting that such mechanisms may nevertheless play an important role in the evolution of cumulative culture in humans.
\end{abstract}

\section{Introduction}

Cultural evolution - change over time in socially learned traits [11, 4] - is ubiquitous in nature. Although humans seem to be unique in the sheer number of traits that we learn from others [34, 20, 30], non-human animals can also acquire and maintain many traits via social learning [31, 3]. Research in cultural evolution therefore often focuses on mechanisms for social 
learning - that is, mechanisms that direct when, what, and from whom to learn [29, 36]. But mechanisms for teaching, which determine what information is available for learners to learn in the first place, are equally important to cultural evolution.

Following Caro and Hauser [8], we take teaching to be a modification in the teacher's behavior that facilitates learning in a naive observer with no immediate benefit (or some cost) to the teacher. Teaching has been found in many non-human animals. In the 1950s, for example, a troop of Japanese macaques (Macaca fuscata) living on the island of Kōjima in Japan were reported to wash sweet potatoes before eating [26]. As potato washing was not observed in other troops, it soon became clear that this skill spread through social learning: after observing a macaque wash a potato before eating it, other macaques were more likely to perform the same behavior in the presence of similar food items. Other skills, such as tool handling, were subsequently shown to likewise spread and persist through social learning among Japanese macaques [24]. Socially transmitted skills have also been documented in other animal species - notable examples include termite fishing in chimpanzees [42, 2], prey handling in meerkats [40], tool use in corvids [25], and foraging patterns among some social insects [15].

Teaching is also a crucial part of social and cultural evolution in humans. The importance of pedagogy is clear in WEIRD societies [21, but a vast array of pedagogical practices has also been documented in hunter-gatherers and other non-Western populations [22, 23, 32, 5, 28]. Indeed, Sterelny [39] proposes that a variety of teaching mechanisms have long been central to the human ecological niche: for their cooperative foraging style to thrive in a changing environment, our ancestors had to develop efficient ways to gather and share information in social groups. In a similar vein, Kline [27] provides a rich taxonomy of mechanisms - from social tolerance and opportunity provisioning to evaluative feedback and direct instruction - that facilitate teaching across species, especially in the case of humans.

In the past decade or so, cognitive scientists have made a great deal of progress in understanding the mechanisms that underlie the capacity for teaching. For example, empirical and theoretical work on pedagogical reasoning has shed light on the cognitive faculties that allow humans to actively share vast amounts of information with others [16, 37, 38]. Research in evolutionary biology has also made important contributions, identifying conditions under which we should expect mechanisms for teaching to evolve in both humans and non-human animals [41, 10, 9, 14]. 
When pursued in isolation, however, both areas of research face serious limitations. Studies in cognitive science are rarely cast in evolutionary terms, taking the mental faculties necessary for information sharing as a given. Conversely, work on the evolution of teaching often proceeds on the basis of formal models that abstract away from the intricacies of animal cognition. But to paint a full picture of how teaching shapes cultural evolution, we must take into account a wide range of cognitive mechanisms and explain how they first came into being. What we need is therefore a multi-pronged approach that integrates aspects of cognition and evolutionary theory. However, to date no model has been proposed to represent both cognitive and evolutionary aspects of teaching.

To remedy this issue, we propose here a simple model that integrates cognitive and evolutionary aspects of teaching. Our model represents a dualinheritance process: individuals acquire and share information about valuable cultural traits at the same time that their capacity to share information about such traits with others is under control of genes that evolve by natural selection. Our model therefore allows us to simultaneously study the cognitive faculties that transmit cultural traits and the evolutionary dynamics that shape these cognitive faculties - for a discussion of strengths and limitations of similar modeling techniques, see Lewens [33].

The paper proceeds as follows. In the next section, we present a simple model of teaching in the context of skill transmission. Individuals can share information about the skills that they possess by choosing data pointsthat is, by choosing to perform a certain behavior in a given environment. In Section 3, we then show that teaching can give rise to an opportunity cost that goes beyond any direct cost that it may also entail. In Section 4, we discuss how this opportunity cost can cause teaching mechanisms to be self-limiting, suggesting that such mechanisms may nevertheless play an important role in the evolution of cumulative culture in humans.

\section{Model}

\section{$2.1 \quad$ Skills}

To represent the transmission of socially learnt skills, our model considers a finite number of environmental states $e=\left\{e_{1}, \ldots, e_{m}\right\}$, a finite number of observable behaviors $b=\left\{b_{1}, \ldots, b_{n}\right\}$, and a finite number of skills 
$s=\left\{s_{1}, \ldots, s_{o}\right\}$. We take a skill to be a disposition to perform a certain behavior in a given environmental state. That is, a skill corresponds to a set of possible data points $d=\left\{d_{1}, \ldots, d_{m \times n}\right\}$, where each data point is given by $d_{i}=\left(e_{j}, b_{k}\right)$ and represents the disposition to perform a certain behavior $\left(b_{k}\right)$ in a particular environment $\left(e_{j}\right)$. More generally, a skill can be represented by a probability distribution over such data points. A skill therefore disposes its bearer to perform a single behavior in a given environmental state, but each skill may be associated with a different behavior depending on the environment.

Our model can represent skills such as potato washing among Japanese macaques. In this case, the presence or absence of a potato represents different environmental states, washing or not washing the food item corresponds to different behaviors, and pairings such as (wash, potato) or (don't wash, potato) are data points. Skills consist in a set of data points - e.g., the disposition to perform the washing behavior or not in the presence of a given food item. In other words, individuals with the skill of potato washing have the disposition to perform the washing behavior in the presence of a sweet potato but not otherwise. Naive individuals who do not possess the skill are not disposed to wash any food item.

As another example, consider prey handling in meerkats. In this case, the presence of harmless or potentially dangerous invertebrates represent different environmental states, disabling the prey or not correspond to different behaviors, and pairings such as (disable prey, scorpion) or (disable prey, beetle) are data points. Skills again consist in a set of data points - the disposition to disable the prey or not before consumption. Skillful individuals thus have the disposition to disable preys that are potentially dangerous but not otherwise, whereas naive individuals may not have the disposition to disable any prey or try to disable all prey.

\subsection{Learning}

For simplicity, we assume that individuals can be either skillful or naive: skillful individuals have the disposition to perform a certain behavior in a given environmental state, and naive individuals do not have that disposition. We also assume that naive individuals use Bayes' rule to learn from skillful individuals. That is, naive individuals learn a skill by updating the probability that they assign to the skill conditional on observing a given data point. In other words, learning takes place according to the following rule: 


$$
P^{L}\left(s_{\ell} \mid d_{i}\right)=\frac{P^{T}\left(d_{i} \mid s_{\ell}\right) P\left(s_{\ell}\right)}{\sum_{\ell=1}^{o} P^{T}\left(d_{i} \mid s_{\ell}\right) P\left(s_{\ell}\right)},
$$

where $d_{i}$ represents a data point given by $d_{i}=\left(e_{j}, b_{k}\right), P\left(s_{\ell}\right)$ is the prior probability that skillful and naive individuals assign to skill $s_{\ell}, P^{L}\left(s_{\ell} \mid d_{i}\right)$ is the probability that the naive individual assigns to the skill upon observing data point $d_{i}$, and $P^{T}\left(d_{i} \mid s_{\ell}\right)$ is the probability that an individual with skill $s_{\ell}$ chooses data point $d_{i}$ and thus the probability that the skillful individual performs behavior $b_{k}$ in environment $e_{j}$. We assume that the prior probability distribution over skills is common to both skillful and naive individuals as we take it to be part of their innate cognitive makeup, with priors representing how much data a naive individual would have to observe before acquiring a given skill. When $P\left(s_{\ell}\right)=0$, a naive individual would therefore not acquire the skill regardless of how much data they observed; when $P\left(s_{\ell}\right)=1$, on the other hand, a naive individual would acquire the skill without observing any data so that in this case the trait may be better thought of as innate.

\subsection{Teaching}

In our model, naive individuals must gather information to learn a skill. At the same time, skillful individuals can share information to facilitate learning. Following Shafto et al. [37, 38], a crucial assumption we make in our model is that skillful individuals share information about their skill by choosing data points. That is, skillful individuals impart their skill to others by showing what behavior to perform in a given environment. To represent a wide range of teaching mechanisms, we assume that skillful individuals choose a data point - that is, what behavior to perform in a given environment - according to the following rule:

$$
P^{T}\left(d_{i} \mid s_{\ell}\right) \propto\left(P^{L}\left(s_{\ell} \mid d_{i}\right)\right)^{\alpha},
$$

where $P^{T}\left(d_{i} \mid s_{\ell}\right)$ is again the probability that the skillful individual assigns to data point $d_{i}, P^{L}\left(s_{\ell} \mid d_{i}\right)$ is the probability that the naive individual assigns to the skill upon observing the data point, and $\alpha$ is what we call the "teaching effort" (a parameter that controls the mode of teaching). This expression states that skillful individuals choose data points with a probability that is proportional not only to their teaching effort, but also to the probability that 
a naive individual would assign to the skill if they were to observe the data point.

Equations 1 and 2 fully describe the learning process. To see how the model works, we can substitute equation 1 into the right-hand side of expression 2. We then normalize the expression so that the probabilities fall in the unit interval. The probability with which a skillful individual chooses a particular data point conditional on their skill is then given by:

$$
P^{T}\left(d_{i} \mid s_{\ell}\right)=\frac{\left(\frac{P^{T}\left(d_{i} \mid s_{\ell}\right) P\left(s_{\ell}\right)}{\sum_{\ell=1}^{o} P^{T}\left(d_{i} \mid s_{\ell}\right) P\left(s_{\ell}\right)}\right)^{\alpha}}{\sum_{i=1}^{m \times n}\left(\frac{P^{T}\left(d_{i} \mid s_{\ell}\right) P\left(s_{\ell}\right)}{\sum_{\ell=1}^{o} P^{T}\left(d_{i} \mid s_{\ell}\right) P\left(s_{\ell}\right)}\right)^{\alpha}},
$$

where the numerator gives the probability that a learner assigns to the skill upon observing a data point (raised to the power of the teachers' effort) and the denominator is simply the sum of this quantity over all data points, thus serving as a normalizing constant. This is a very general rule for teaching, stating that a skillful individual can take into account the learner's update rule when choosing what data point to produce. Depending on $\alpha$, the skillful individual may also increase or decrease the probability with which they display a particular behavior in a given environment or control in what environment to perform a given behavior so as to modulate the learner's response.

For our purposes, the main quantity of interest is $\alpha$ - the teaching effort. Different values of $\alpha$ represent different teaching mechanisms. When $\alpha=$ 0 , the skillful individual simply chooses what data to produce at random from the set of all possible data points. This means that the individual does not take into account how the learner will learn from the data, nor do they use their skill for their own benefit. In what follows, we therefore do not consider the case of $\alpha=0$ as we are interested in cases where skillful individuals actually use their skills, either to share information with others or for their own benefit. When $\alpha \rightarrow 0$, the skillful individual chooses data points at random from the set of all data points that are consistent with the skill. When $\alpha=1$, the skillful individual chooses data points in direct proportion to the posterior probability that a Bayesian learner would assign to the skill in question if the learner were to observe the data point. The skillful individual therefore not only chooses data points consistent with their skill, but also takes into account how the learner will learn from the data. In the limiting case where $\alpha \rightarrow \infty$, the skillful individual chooses data points so as to maximize the posterior probability that the learner would assign to 
the skill if the learner were to observe the data.

For simplicity, we assume here that environmental states and behaviors are discrete. This means that when $\alpha=0$, the probability of choosing a particular data point given any skill is $\frac{1}{m \times n}$, where $m \times n$ is the number of all possible data points. When $\alpha \rightarrow 0$, the probability of choosing data point $d_{i}$

given skill $s_{\ell}$ is equal to $\frac{1}{\left|s_{\ell}\right|}$, where $\left|s_{\ell}\right|$ is the number of data points that are consistent with skill $s_{\ell}$. And when $\alpha=1$, the probability of choosing a data point depends on how a learner following equation 1 would learn. Equation 3 is therefore a very general way to represent a variety of teaching mechanisms, with the teaching effort $\alpha$ determining how helpful to the learner the teaching mechanism will be.

\subsection{An example}

To illustrate, consider the following example. Suppose that there are two possible behaviors that we represent by $b=\left\{b_{1}, b_{2}\right\}$. Suppose also that there are four environmental states given by $e=\left\{e_{1}, e_{2}, e_{3}, e_{4}\right\}$. There are therefore eight possible data points, as each behavior may be performed in four different environments. Now suppose that there are two skills given by $s=\left\{s_{1}, s_{2}\right\}$. Suppose further that data points $d_{1}=\left(e_{1}, b_{1}\right), d_{4}=\left(e_{4}, b_{1}\right)$, $d_{6}=\left(e_{2}, b_{2}\right)$, and $d_{7}=\left(e_{3}, b_{2}\right)$ are consistent with skill $s_{1}$ but that the remaining data points are not. Similarly, suppose that $d_{1}=\left(e_{1}, b_{1}\right), d_{2}=$ $\left(e_{2}, b_{1}\right), d_{7}=\left(e_{3}, b_{2}\right)$, and $d_{8}=\left(e_{4}, b_{2}\right)$ are consistent with skill $s_{2}$ but that all the other ones are not. Skills $s_{1}$ and $s_{2}$ are depicted in Figure 1; see also Table 1. If a skill assigns a behavior in a given environmental state, the probability of that behavior given that state is 1 ; if the skill does not assign that behavior to the state, the probability of that behavior given the state is 0 . Skills $s_{1}$ and $s_{2}$ therefore coincide in environmental states $e_{1}$ and $e_{3}$, but come apart in $e_{2}$ and $e_{4}$.

To complete our model specification, we need to define initial likelihoods. That is, we need to define a probability distribution over data points conditional on a particular skill independently of the learner's behavior. This conditional distribution represents an individual's disposition to choose data points independently of how the learner would learn from the data. Although in principle any distribution will do, a plausible assumption to make is that in this case an individual samples at random from the set of data points that are consistent with their skill. In our example above, this means that the initial likelihoods given skill $s_{1}$ are uniformly distributed over the data 


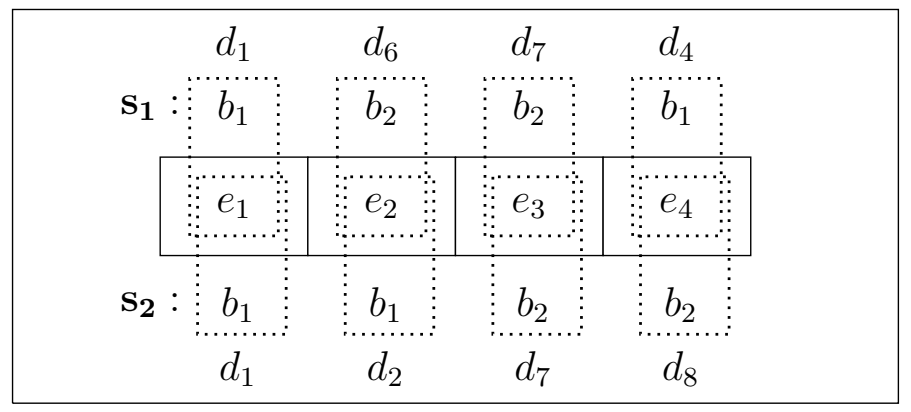

Figure 1: Example with two skills. Skills assign behaviors to environments, represented by the internal rectangles. Each behavior-environment pair consists in a data point, as indicated by the dotted rectangles (blue for data points belonging to $s_{1}$ and red for $s_{2}$ ). Note that a data point might belong to one skill only, both skills, or none at all. For example, data point $d_{1}$, corresponding to pair $\left(e_{1}, b_{1}\right)$, belongs to both skills, while $d_{4}$ only belongs to $s_{1}$. See Table 1 for a full accounting of data points in this example.

Table 1: Data points for two skills. List of all available data points, the corresponding behavior-environment pair, and the skills that are consistent with them.

\begin{tabular}{ccc||ccc}
$d_{i}$ & $\left(e_{j}, b_{k}\right)$ & $s_{\ell}$ & $d_{i}$ & $\left(e_{j}, b_{k}\right)$ & $s_{\ell}$ \\
\hline$d_{1}$ & $\left(e_{1}, b_{1}\right)$ & $s_{1}, s_{2}$ & $d_{5}$ & $\left(e_{1}, b_{2}\right)$ & - \\
$d_{2}$ & $\left(e_{2}, b_{1}\right)$ & $s_{2}$ & $d_{6}$ & $\left(e_{2}, b_{2}\right)$ & $s_{1}$ \\
$d_{3}$ & $\left(e_{3}, b_{1}\right)$ & - & $d_{7}$ & $\left(e_{3}, b_{2}\right)$ & $s_{1}, s_{2}$ \\
$d_{4}$ & $\left(e_{4}, b_{1}\right)$ & $s_{1}$ & $d_{8}$ & $\left(e_{4}, b_{2}\right)$ & $s_{2}$
\end{tabular}

points that are consistent with $s_{1}$ so that $P\left(d_{i} \mid s_{1}\right)=0.25$ for $i=1,4,6,7$ and $P\left(d_{j} \mid s_{1}\right)=0$ for $j \neq i$. Similarly, the initial likelihoods for skill $s_{2}$ are $P\left(d_{i} \mid s_{2}\right)=0.25$ for $i=1,2,7,8$ and $P\left(d_{j} \mid s_{2}\right)=0$ for $j \neq i$.

Second, we need the final likelihoods with which a skillful individual chooses data points when sharing information with a learner. That is, we need a probability distribution over data points conditional on a particular skill that takes into account the teaching mechanism given by equation 3 . Given initial likelihoods, priors, and a particular value of $\alpha$, we can derive the distribution of final likelihoods by solving equation 3 through fixed-point iteration. To iterate to a fixed point, we start by plugging the initial likelihood of a data point together with the remaining parameters into the right-hand 
side of the equation. Next, we take the likelihood value just obtained in the left-hand side of the equation and use it as the likelihood that goes into the right-hand side of the equation in the next iteration. We then iterate this procedure until the likelihood value obtained in the left-hand side of the equation equals the likelihood value used in the right-hand side of the equation. This value is a fixed point. Finding the fixed points for the equation given the initial likelihoods for all the data points gives us the final likelihoods - that is, the probability with which a skillful individual chooses data points when taking the learner's behavior into account and exerting a certain teaching effort (see Figure 2).

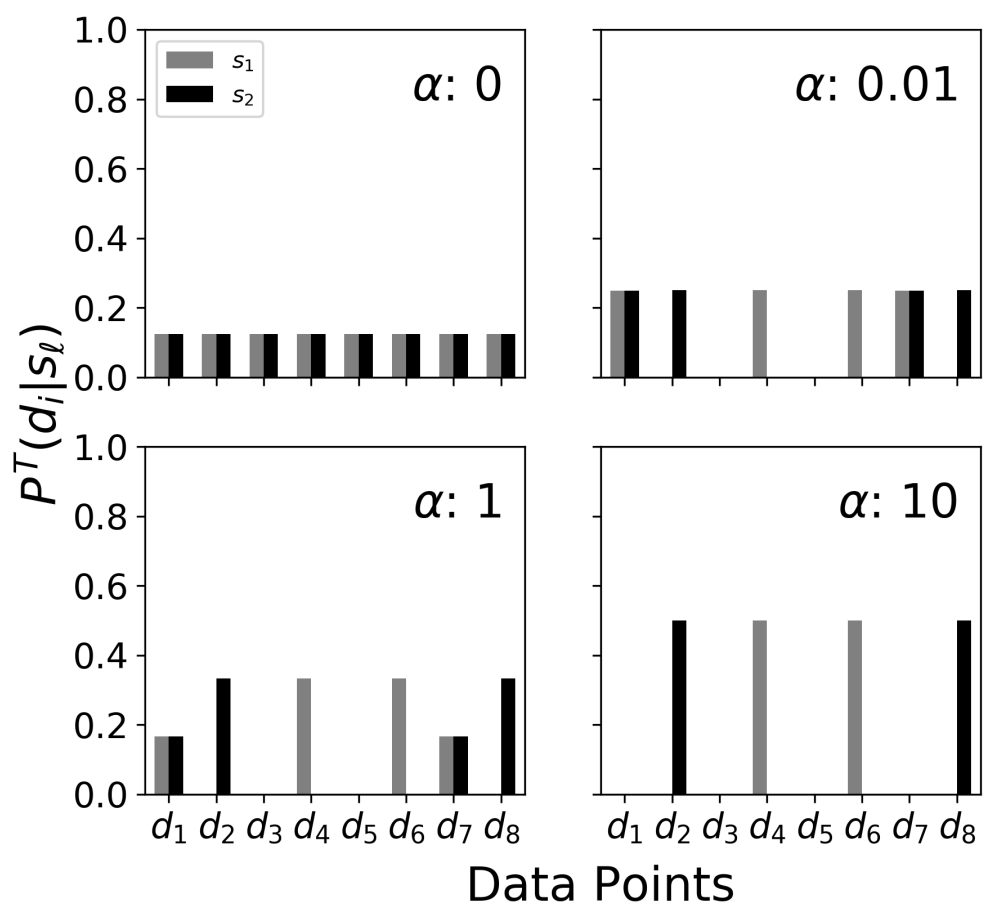

Figure 2: Example with two skills. Final likelihoods given different values of $\alpha$ in the example depicted in Figure 1. Initial likelihoods are given by random sampling of consistent data points. Higher values of $\alpha$ favor sampling the data points that are most helpful for the learner. The prior distributions over skills is uniform. 


\section{$2.5 \quad$ Fitness}

Whereas skills spread through social learning, we assume in our model that teaching effort is under genetic control. This means that skills evolve at a much shorter timescale than teaching effort, so that a novel and beneficial skill will reach its equilibrium frequency in the population long before natural selection can act on the genes that control teaching effort. We also assume that teaching effort is subject to multiple small-effect genes, so that it varies continuously. We therefore consider how teaching effort affects a continuous fitness function when the frequency of skillful and naive individuals in the population is held constant.

In particular, we suppose that the fitness of skillful individuals takes the following form. Skillful individuals receive a payoff $\pi_{i}$ for performing behavior $b_{k}$ in environment $e_{j}$. In other words, skillful individuals receive a payoff $\pi_{i}$ for choosing data point $d_{i}=\left(e_{j}, b_{k}\right)$. The direct fitness gain to the skillful individual is then given by $\sum_{i=1}^{m \times n} P^{T}\left(d_{i} \mid s_{\ell}\right) \pi_{i}$, which is the expected payoff for all data points. As per expression 3 , the term $P^{T}\left(d_{i} \mid s_{\ell}\right)$ takes into account the skillful individual's mode of teaching. So the direct fitness gain to the skillful individual depends on the teaching effort $\alpha$.

By observing a skillful individual's choice of data point, a learner can then acquire the skill. Once learners acquire the skill, however, they need not behave exactly as the individual from whom they acquired the skill. This is because we assume that the teaching mechanism of skillful individuals takes the form given in equation 3, so that depending on teaching effort skillful individuals may consider the learners' behavior when choosing a data point. But we assume that learners do not do the same: learners behave in accordance with their newly acquired skill across different environments, but they do not take into account how other learners would learn from the data points that they choose to produce.

To represent this, we let $\bar{\pi}_{\ell}$ be the payoff that the learner expects to receive after acquiring skill $s_{\ell}$. If learners sample at random from the set of data points consistent with skill $s_{\ell}$, we have $\bar{\pi}_{\ell}=\sum_{i=1}^{\left|s_{\ell}\right|} \pi_{i} \frac{1}{\left|s_{\ell}\right|}$.

Finally, we let $r$ represent the coefficient of relatedness between skillful and naive individuals. This parameter represents the degree to which skillful individuals have an interest in the evolutionary fate of naive individuals. In other words, skillful individuals get a fraction $r$ of the payoff that naive individuals receive from learning the skill. We also assume that skillful individuals incur a direct cost $c(\alpha)$ that is function of their teaching effort $\alpha$, 
representing the cognitive or metabolic cost of taking the learners' behavior into account. Assuming that teaching bears a direct cost, we let $c(\alpha)$ be an increasing function of the sharing effort.

Putting all this together, we now let $w(\alpha)$ represent the fitness of skillful individuals as a function of their teaching effort:

$$
w(\alpha)=\underbrace{\sum_{i=1}^{m \times n} P^{T}\left(d_{i} \mid s_{\ell}\right) \pi_{i}}_{\text {direct benefit }}+\underbrace{r \bar{\pi}_{\ell} \sum_{i=1}^{m \times n} P^{T}\left(d_{i} \mid s_{\ell}\right) P^{L}\left(s_{\ell} \mid d_{i}\right)}_{\text {indirect benefit }}-\overbrace{c(\alpha)}^{\text {direct cost }}
$$

where the first term in the right-hand side of this equation represents the direct fitness benefit that skillful individuals accrue from behaving in accordance with their skill, the second term represents the indirect fitness benefit that skillful individuals receive from learners acquiring the skill scaled by the relatedness between them, and the third term represents the direct cost of teaching.

When the final likelihoods, priors, and payoffs are known, it is possible to calculate the fitness of skillful individuals for any given value of $r$ and $\alpha$. This means we can study the evolution of teaching by analyzing the behavior of the teaching effort $\alpha$ in equation 4 under different degrees of relatedness. We can thus determine the conditions under which natural selection should or should not favor the spread of teaching mechanism. In the next section, we state a few results.

\section{Results}

We start by considering the fitness of skillful individuals who exert minimal sharing effort, i.e., $\alpha \rightarrow 0$. From equation (4), the fitness of skillful individuals who exert minimal sharing effort is given by:

$$
w_{\text {null }}=\bar{\pi}_{\ell}+r \bar{\pi}_{\ell} P_{\text {null }} .
$$

where $P_{\text {null }}=\sum_{i=1}^{m \times n} P^{T}\left(d_{i} \mid s_{\ell}\right) P^{L}\left(s_{\ell} \mid d_{i}\right)$ is simply the probability that naive individuals acquire the skill in the absence of any teaching effort on the part of skillful individuals. We call expression (5) the "baseline fitness" of skillful individuals. This allows us to write our first result, which is a version of Hamilton's rule for sharing effort. 
Result 1. If $\pi_{i}$ is a constant $k$ for all data points $d_{i}$, skillful individuals who exert positive teaching effort can invade a population of skillful individuals where teaching effort is absent provided that $r b(\alpha)>c(\alpha)$, where $b(\alpha)=\bar{\pi}_{\ell}\left(\sum_{i=1}^{m \times n} P^{T}\left(d_{i} \mid s_{\ell}\right) P^{L}\left(s_{\ell} \mid d_{i}\right)-P_{n u l l}\right)$ is the increase in the indirect fitness benefit experienced by skillful individuals who exert positive teaching effort.

Skillful individuals who exert positive teaching effort can invade a population of skillful individuals with minimal teaching effort provided that the following condition is met:

$$
w_{\text {null }}<w(\alpha)
$$

for an $\alpha>0$.

To determine the conditions under which inequality (6) holds, we start by observing that the direct fitness benefit that skillful individuals receive from behavior that is consistent with their skill is $\sum_{i=1}^{m \times n} P^{T}\left(d_{i} \mid s_{\ell}\right) \pi_{i}=\bar{\pi}_{\ell}=k$ no matter what value of $\alpha$. This is because, by hypothesis, $\pi_{i}$ takes a constant value $k$ for all data points $d_{i}$. For the same reason, the fitness benefit that naive individual receive from learning the skill is also $\bar{\pi}_{\ell}=k$. The fitness of skillful individuals who exert positive teaching effort is therefore:

$$
w(\alpha)=k+r k \sum_{i=1}^{m \times n} P^{T}\left(d_{i} \mid s_{\ell}\right) P^{L}\left(s_{\ell} \mid d_{i}\right)-c(\alpha) .
$$

For short, we can write $\bar{\pi}_{\ell}\left(\sum_{i=1}^{m \times n} P^{T}\left(d_{i} \mid s_{\ell}\right) P^{L}\left(s_{\ell} \mid d_{i}\right)-P_{\text {null }}\right)$ as $b(\alpha)$. This is the increase in the indirect fitness benefit that skillful individuals who exert positive teaching effort accrue from the behavior of learners who acquire the skill relative to the baseline indirect fitness benefit of skillful individuals who do not exert any teaching effort. With constant payoffs, condition (6) is now given by $k+r k P_{\text {null }}<k+r k \sum_{i=1}^{m \times n} P^{T}\left(d_{i} \mid s_{\ell}\right) P^{L}\left(s_{\ell} \mid d_{i}\right)-c(\alpha)$. Re-arranging this expression, the condition for mutants who exert positive teaching effort to invade a population with no teaching effort therefore holds when:

$$
r b(\alpha)>c(\alpha) .
$$

This condition states that natural selection favors an increase in teaching effort from a baseline with no teaching effort when the increase in the indirect fitness benefit that skillful individuals who exert positive teaching effort 
receive from naive individuals acquiring the skill, scaled by the degree of relatedness between them, is greater than the direct cost of teaching naive individuals. The condition for positive teaching effort to invade therefore takes the form of Hamilton's rule $(1964 \mathrm{a}, 1964 \mathrm{~b})$. This is not altogether surprising, as we assumed that there is a direct cost $(c(\alpha))$ to teaching and a direct benefit to the naive individuals, making our teaching mechanisms a kind of altruistic behavior Thornton and Raihani [41], Fogarty et al. [14].

Next, we show that teaching can be altruistic even in the absence of direct cost. When payoffs are not constant across data points, there can be a tradeoff between the direct and the indirect fitness gain that skillful individuals receive. In this case, the evolution of teaching can be self-limiting regardless of the direct cost that any sharing effort would entail simply because there is an opportunity cost that is inherent to teaching.

Result 2. If $\pi_{i}$ is not constant across all data points $d_{i}$, skillful individuals who exert a positive teaching effort can invade a population of skillful individuals where teaching is absent provided that $r b(\alpha)>c_{o p}(\alpha)$, where $c_{o p}(\alpha)$ is the opportunity cost of teaching and it is given by $c_{o p}(\alpha)=\bar{\pi}_{\ell}-\pi(\alpha)+c(\alpha)$ with $\bar{\pi}_{\ell}$ defined as before and $\pi(\alpha)=\sum_{i}^{m \times n} P^{T}\left(d_{i} \mid s_{\ell}\right) \pi_{i}$ is the payoff for skillful individuals who exert positive teaching effort $\alpha$.

To show this result, we again compare the fitness of skillful individual who exert positive teaching effort with the baseline fitness. With payoffs that are not constant, the fitness of skillful individuals with teaching effort $\alpha>0$ is given by:

$$
w(\alpha)=\pi(\alpha)+r \bar{\pi}_{\ell} \sum_{i=1}^{m \times n} P^{T}\left(d_{i} \mid s_{\ell}\right) P^{L}\left(s_{\ell} \mid d_{i}\right)-c(\alpha)
$$

where $\pi(\alpha)=\sum_{i}^{m \times n} P^{T}\left(d_{i} \mid s_{\ell}\right) \pi_{i}$ is the direct fitness benefit that skillful individuals receive from their skill when exerting teaching effort $\alpha$.

According again to condition (6), skillful individuals who exert positive teaching effort can invade a population of skillful individuals where teaching is absent provided that:

$$
w_{n u l l}<\pi(\alpha)+r \bar{\pi}_{\ell} \sum_{i=1}^{m \times n} P^{T}\left(d_{i} \mid s_{\ell}\right) P^{L}\left(s_{\ell} \mid d_{i}\right)-c(\alpha)
$$


where $w_{\text {null }}$ is again given by equation (5) and all other quantities are defined as before.

Again, we can write $b(\alpha)$ for $\bar{\pi}_{\ell}\left(\sum_{i=1}^{m \times n} P^{T}\left(d_{i} \mid s_{\ell}\right) P^{L}\left(s_{\ell} \mid d_{i}\right)-P_{\text {null }}\right)$. We can also write $\bar{\pi}_{\ell}-\pi(\alpha)+c(\alpha)$ as $c_{o p}(\alpha)$, which is the opportunity cost of teaching. The opportunity cost is the difference between the direct fitness benefit to skillful individuals from behavior that is simply consistent with their skill and the direct fitness benefit from behaving in accordance with their skill at teaching effort $\alpha$, plus the direct cost of that teaching effort. Substituting $b(\alpha)$ and $c_{o p}(\alpha)$ into equation $(10)$ and re-arranging it, we obtain our result:

$$
r b(\alpha)>c_{o p}(\alpha)
$$

This condition resembles condition (8) in stating that natural selection favors an increase in teaching effort when the indirect fitness benefit that skillful individuals receive from naive individuals acquiring the skill, scaled by the degree of the relatedness between them, is greater than the cost of teaching naive individuals. On the other hand, it differs from condition (8) in that the relevant cost is not simply the direct cost due to teaching effort. It is rather the opportunity cost of teaching naive individuals when behavior that is consistent with the relevant skill yields a higher payoff. The contrast is especially salient when $c(\alpha)$ is negligible. In this case, condition (8) can be met even when condition (11) is not. If this opportunity cost is sufficiently high, condition (11) says that natural selection limits the spread of teaching even when the direct cost of information sharing is null.

It is also possible to show that under some very liberal conditions natural selection favors a positive but finite teaching effort.

Result 3. If $\pi_{i}$ is not constant across all data points $i$, then skillful individuals who exert positive but finite teaching effort can invade a population of skillful individuals with perfect teaching provided that the direct cost of teaching $c(\alpha)$ is a strictly increasing function of $\alpha$.

To prove this result, we start by calculating the fitness of skillful individuals when $\alpha \rightarrow \infty$. We call this the "upper-bound effort" of teaching. The fitness of skillful individuals exerting the upper-bound effort is given by: 


$$
w_{u p}=\pi_{u p}+r \bar{\pi}_{\ell} \sum_{i=1}^{m \times n} P^{T}\left(d_{i} \mid s_{\ell}\right) P^{L}\left(s_{\ell} \mid d_{i}\right)-c_{u p} .
$$

where $\pi_{u p}$ and $c_{u p}$ are the direct fitness benefit and the direct cost to skillful individuals when their teaching effort goes to infinity. In this limiting case, we assume that learning is as effective as it can be since skillful individuals exert infinite teaching effort. This means that $\sum_{i}^{m \times n} P^{T}\left(d_{i} \mid s_{\ell}\right) P^{L}\left(s_{\ell} \mid d_{i}\right) \rightarrow 1$ as $\alpha \rightarrow \infty$. With this simplifying assumption, we can re-write expression 12 as:

$$
w_{u p}=\pi_{u p}+r \bar{\pi}_{\ell}-c_{u p} \quad .
$$

According again to condition (6), skillful individuals who exert a positive but finite teaching effort can therefore invade a population of skillful individuals with infinite teaching effort provided that:

$$
\pi(\alpha)+r \bar{\pi}_{\ell} \sum_{i}^{m \times n} P^{T}\left(d_{i} \mid s_{\ell}\right) P^{L}\left(s_{\ell} \mid d_{i}\right)-c(\alpha)>\pi_{u p}+r \bar{\pi}_{\ell}-c_{u p}
$$

This is a relatively weak requirement, since in many interactions, the direct cost of teaching is likely to grow such that the cost for ensuring that naive individuals learn with certainty is much bigger than a finite effort $c_{u p} \gg$ $c(\alpha)$. This means condition 14 does not pose a very stringent requirement for natural selection to favor a positive but finite teaching effort.

\subsection{An example}

To illustrate our results, consider again the numerical example introduced above. Suppose that the payoffs for data points are not constant so that some data points yield a higher payoff than others (see Table 2). Suppose also that the direct cost that individuals possessing skill $s_{1}$ incur for teaching is given by $c(\alpha)=k \cdot \alpha$. Now suppose that $k=0.05$ so that the direct cost of teaching is very high. In this case, the optimal $\alpha$ is zero for all values of $r$ and thus skillful individuals with a positive $\alpha$ cannot invade a population of individuals exerting no teaching effort (Figure 3 , left).

Now suppose that $k=0.01$ so that the direct cost of teaching is nonnegligible but not very high. In this case, skillful individuals with a positive 
but finite teaching effort can invade a population of skillful individuals exerting no teaching effort, since the optimal $\alpha$ is positive but finite-provided, of course, that $r$ is sufficiently high (Figure 3 , center).

But suppose now that $k=0$ so that the direct cost of teaching is null. In this case, teaching can evolve depending on the value of $r$. When $r=$ 1.0, for example, the fitness of skillful individuals who exert no teaching effort is $w(0)=2.3$. But the fitness of skillful individuals who exert a very high teaching effort (e.g. $\alpha=10$ ) is $w(\alpha)=2.44$. When $r$ is high, it is therefore optimal for skillful individuals to invest as much effort in teaching as possible - provided, of course, that there is no direct cost in doing so. When $r=0$, on the other hand, the fitness of skillful individuals who exert no teaching effort is $w(\alpha)=1.31$. In contrast, the fitness of skillful individuals with $\alpha=10$ is $w(\alpha)=1.12$. When $r$ is low, the best strategy is thus for skillful individuals to abstain from teaching. In this case, the optimal value of $\alpha$ is zero even though the direct cost of teaching is null. This is because of the opportunity cost inherent to teaching (Figure 3, right).

Table 2: Payoffs for two skills. Payoffs for all available data points in the example given in Figure 1 .

\begin{tabular}{ccc||ccc}
$d_{i}$ & $\left(e_{j}, b_{k}\right)$ & $\pi_{i}$ & $d_{i}$ & $\left(e_{j}, b_{k}\right)$ & $\pi_{i}$ \\
\hline$d_{1}$ & $\left(e_{1}, b_{1}\right)$ & 2 & $d_{5}$ & $\left(e_{1}, b_{2}\right)$ & 0 \\
$d_{2}$ & $\left(e_{2}, b_{1}\right)$ & 0.25 & $d_{6}$ & $\left(e_{2}, b_{2}\right)$ & 0.25 \\
$d_{3}$ & $\left(e_{3}, b_{1}\right)$ & 0 & $d_{7}$ & $\left(e_{3}, b_{2}\right)$ & 1 \\
$d_{4}$ & $\left(e_{4}, b_{1}\right)$ & 2 & $d_{8}$ & $\left(e_{4}, b_{2}\right)$ & 0.25
\end{tabular}

\section{Discussion}

The evolution of teaching can radically alter cultural and genetic evolutionary trajectories of social species. Here, we provided a new model that integrates cognitive and evolutionary aspects of information sharing by combining recent work in cognitive science and social evolutionary theory. In this model, we account for the fact that individuals with transmissible skills can behave in ways that can make learners more or less likely to acquire the skill-i.e., by choosing different data points). We consider the evolution of this teaching effort, quantified by the variable $\alpha$ in our model. 

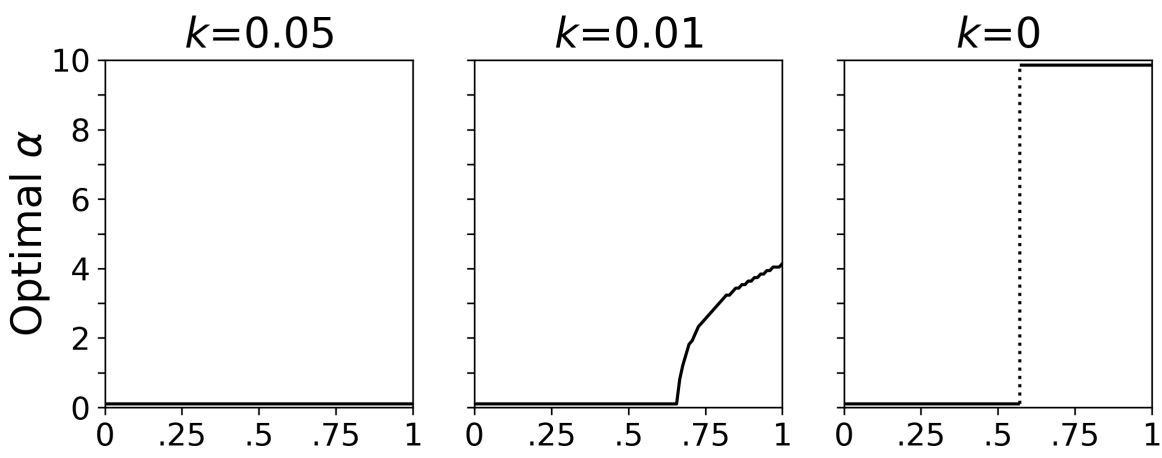

Figure 3: The optimal $\alpha$ depends on $r$ and $k$. When $k=0.05$, the direct cost of teaching is too high and no effort is thus optimal regardless of $r$ (left). When $k=0.01$, the direct cost of teaching takes intermediate values and so a finite effort is optimal for $r^{*}>0.72$ but below this threshold minimal effort is optimal (center). But when $k=0$, skillful individuals do best by either putting no effort $\left(r^{*}<0.61\right)$ or by putting as much effort as possible into teaching $\left(r^{*}>0.61\right)$. This is due to the opportunity cost of teaching (right). Shown are results for payoffs given in Table 2 .

A boundary case of our model is that there is no information contained in the behavior of the skillful individual, which happens when $\alpha=0$. In this boundary case, "skillful" individuals simply choose data points (behaviorenvironment pairs) randomly amongst all possible pairs, and transmit no information. However, this boundary case represents a discontinuity: when $\alpha \rightarrow 0$ (but not exactly zero), skillful individuals' behavior converges to choosing only data points that are compatible with their skill. In other words, skillful individuals with vanishingly small teaching effort will share information on what behavior to perform in a given environment passively, just by executing behavior that is consistent with their skill. In Kline's (2015) taxonomy of teaching mechanisms, this is closest to the case of social tolerance: by tolerating the presence of others and allowing them to observe, skillful individuals teaching simply by carrying out the appropriate behavior. In contrast, when $\alpha>0$, skillful individuals go above and beyond merely performing behavior consistent with their skill. In this case, skillful individuals preferentially provide learners with data that facilitate learning. Examples of this are what Kline calls opportunity provisioning and stimulus enhancement. When $\alpha \geq 1$, skillful individuals chose what data to display with 
probability that is at least proportional to how naive individuals would learn when exposed to the data. In Kline's terminology, this might corresponds to direct forms of teaching, such evaluative feedback and direct teaching.

In our evolutionary model, we assume that skillful individuals get indirect fitness benefits from the increased probability of learning by the learners. In this setting, we can identify conditions under which selection favors the spread of positive teaching effort. Selection favors a higher teaching effort when the relatedness between skillful individuals and naive learners is sufficiently high, sharing costs are sufficiently low, and the benefit of transmitting the skill is sufficiently high. Importantly, our model also identifies two kinds of costs of teaching: the first is the direct cognitive or metabolic cost in taking the informational need of others into account, while the second is the opportunity cost in sharing information instead of exploiting it to increase one's own fitness. We thus show that teaching entails an "explain-exploit" trade-off that is similar to the explore-exploit trade-off in information acquisition [36], adding a new dimension to potential costs of teaching not explicitly considered in previous models [14, 10].

Our model is in keeping with Sterelny's (2012) discussion of the evolutionary trajectory of modern humans. For Sterelny, the first evolutionary step towards the information-rich ecological niche that we came to occupy was the formation of relatively large social groups. By living and foraging in groups, our ancestors could share information about their skills with others simply by tolerating the presence of onlookers. In our model, this corresponds to the case where $\alpha \rightarrow 0$ so that skillful individuals share information with others simply by performing behavior in accordance with their skill. As groups grew more cooperative, large, and cohesive, higher levels of information sharing became possible through opportunity provisioning. This corresponds to the case where $\alpha>0$ and skillful individuals provide learners with higher-quality information. But as Sterelny points out, it was not until the crudest form of communication arose that high-volume and high-fidelity forms of teaching finally spread. This could correspond to $\alpha \geq 1$, as skillful individuals choose what behavior to perform with probability that is at least proportional to how naive individuals would learn when exposed to the behavior. Following Aaby and Ramsey's (2020) tripartite taxonomy, teaching can therefore be understood as a form of "external" niche construction when the skillful individual alters the naive individual's learning environment to serve a pedagogical function.

But our model results also highlight some additional subtleties to Sterelny's 
insight that advances in how our ancestors shared information with one another favored the spread of better information gathering, creating a positive feedback loop between mechanisms for sharing and gathering information. Our model shows that either the direct cost or the opportunity cost of teaching can act as brakes to this feedback loop, limiting the evolution and strength of teaching. More precisely, our model indicates that even when the direct cost of teaching is negligible, teaching can still result in an opportunity cost that threatens to dampen the positive feedback loop between sharing and gathering information. This could help explain why extreme forms of teaching - such as evaluative feedback and direct instruction - are a relatively rare and evolutionarily recent phenomena.

More generally, our model sheds new light on current debates about the role of teaching in human cultural evolution. For all the similarities between human and non-human culture, human culture seems to be unique in that high-fidelity and high-volume social learning leads to a series of adaptations that gradually build on one another. This is what many now term "cumulative culture", though the concept remains contentious - see Mesoudi and Thornton [35] for a review and discussion. To explain the cumulative character of human culture, some invoke our capacity for teaching [6, 7, 13]. Others disagree, denying that teaching could play such a prominent role in cumulative cultural evolution [43, 12].

However, both camps in this debate seem to be narrowly concerned with a very particular and extreme forms of teaching: teaching via evaluative feedback or direct instruction. In contrast, our model highlights that it is possible to teach others without direct teaching as teaching effort comes in degrees. In fact, it is likely that human culture benefits especially from our capacity to teach in a variety of different forms. Both sides in the debate about the importance of teaching in cumulative culture might therefore do well to consider different forms of teaching, especially less extreme ones.

In conclusion, by linking the emergence of cumulative culture with the cognitive basis of information sharing in humans, our model therefore blends different "grains of analysis" in the study of cultural change [17]. In doing so, our model helps advance the synthesis between micro- and macro-scale processes in cultural evolution in the context of the evolution of teaching [34]. Continuing this synthesis will likely reveal more interesting insights on the evolution, functioning, and consequences of teaching. 


\section{References}

[1] Aaby, B. H. and Ramsey, G. (2020). Three kinds of niche construction. The British Journal for the Philosophy of Science, 0:1-25.

[2] Boesch, C., Kalan, A. K., Mundry, R., Arandjelovic, M., Pika, S., Dieguez, P., Ayimisin, E. A., Barciela, A., Coupland, C., Egbe, V. E., et al. (2020). Chimpanzee ethnography reveals unexpected cultural diversity. Nature Human Behaviour, pages 1-7.

[3] Bonner, J. T. (2018). The evolution of culture in animals. Princeton University Press.

[4] Boyd, R. and Richerson, P. J. (1988). Culture and the evolutionary process. University of Chicago Press.

[5] Boyette, A. H. and Hewlett, B. S. (2018). Teaching in hunter-gatherers. Review of Philosophy and Psychology, 9(4):771-797.

[6] Burdett, E. R., Dean, L. G., and Ronfard, S. (2018). A diverse and flexible teaching toolkit facilitates the human capacity for cumulative culture. Review of Philosophy and Psychology, 9(4):807-818.

[7] Caldwell, C. A., Renner, E., and Atkinson, M. (2018). Human teaching and cumulative cultural evolution. Review of Philosophy and Psychology, $9(4): 751-770$.

[8] Caro, T. M. and Hauser, M. D. (1992). Is there teaching in nonhuman animals? The Quarterly Review of Biology, 67(2):151-174.

[9] Castro, L., Castro-Nogueira, M. Á., Villarroel, M., and Toro, M. Á. (2019). The role of assessor teaching in human culture. Biological Theory, $14(2): 112-121$.

[10] Castro, L. and Toro, M. A. (2014). Cumulative cultural evolution: The role of teaching. Journal of Theoretical Biology, 347:74-83.

[11] Cavalli-Sforza, L. L. and Feldman, M. W. (1981). Cultural transmission and evolution: A quantitative approach. Princeton University Press. 
[12] de Oliveira, E., Reynaud, E., and Osiurak, F. (2019). Roles of technical reasoning, theory of mind, creativity, and fluid cognition in cumulative technological culture. Human Nature, 30(3):326-340.

[13] Dean, L. G., Kendal, R. L., Schapiro, S. J., Thierry, B., and Laland, K. N. (2012). Identification of the social and cognitive processes underlying human cumulative culture. Science, 335(6072):1114-1118.

[14] Fogarty, L., Strimling, P., and Laland, K. N. (2011). The evolution of teaching. Evolution: International Journal of Organic Evolution, 65(10):2760-2770.

[15] Franklin, E. L. and Franks, N. R. (2012). Individual and social learning in tandem-running recruitment by ants. Animal behaviour, 84(2):361-368.

[16] Gergely, G., Egyed, K., and Király, I. (2007). On pedagogy. Developmental science, 10(1):139-146.

[17] Godfrey-Smith, P. (2012). Darwinism and cultural change. Philosophical Transactions of the Royal Society B: Biological Sciences, 367(1599):21602170 .

[18] Hamilton, W. D. (1964a). The genetical evolution of social behaviour. I. Journal of theoretical biology, $7(1): 1-16$.

[19] Hamilton, W. D. (1964b). The genetical evolution of social behaviour. II. Journal of theoretical biology, 7(1):17-52.

[20] Henrich, J. (2017). The secret of our success: How culture is driving human evolution, domesticating our species, and making us smarter. Princeton University Press.

[21] Henrich, J., Heine, S. J., and Norenzayan, A. (2010). The weirdest people in the world? Behavioral and brain sciences, 33(2-3):61-83.

[22] Hewlett, B. S., Fouts, H. N., Boyette, A. H., and Hewlett, B. L. (2011). Social learning among congo basin hunter-gatherers. Philosophical Transactions of the Royal Society B: Biological Sciences, 366(1567):1168-1178.

[23] Hewlett, B. S. and Roulette, C. J. (2016). Teaching in hunter-gatherer infancy. Royal Society Open Science, 3(1):150403. 
[24] Huffman, M. A. and Quiatt, D. (1986). Stone handling by japanese macaques (macaca fuscata): implications for tool use of stone. Primates, 27(4):413-423.

[25] Hunt, G. R. (1996). Manufacture and use of hook-tools by new caledonian crows. Nature, 379(6562):249-251.

[26] Kawamura, S. (1959). The process of sub-culture propagation among japanese macaques. Primates, 2(1):43-60.

[27] Kline, M. A. (2015). How to learn about teaching: An evolutionary framework for the study of teaching behavior in humans and other animals. Behavioral and Brain sciences, 38.

[28] Kline, M. A., Boyd, R., and Henrich, J. (2013). Teaching and the life history of cultural transmission in fijian villages. Human Nature, 24(4):351374 .

[29] Laland, K. N. (2004). Social learning strategies. Animal Learning 83 Behavior, 32(1):4-14.

[30] Laland, K. N. (2018). Darwin's unfinished symphony: How culture made the human mind. Princeton University Press.

[31] Laland, K. N. and Galef, B. G. (2009). The question of animal culture. Harvard University Press.

[32] Lew-Levy, S., Kissler, S. M., Boyette, A. H., Crittenden, A. N., Mabulla, I. A., and Hewlett, B. S. (2020). Who teaches children to forage? exploring the primacy of child-to-child teaching among hadza and bayaka hunter-gatherers of tanzania and congo. Evolution and Human Behavior, $41(1): 12-22$.

[33] Lewens, T. (2015). Cultural evolution: conceptual challenges. OUP Oxford.

[34] Mesoudi, A. (2011). Cultural evolution: How Darwinian theory can explain human culture and synthesize the social sciences. University of Chicago Press. 
[35] Mesoudi, A. and Thornton, A. (2018). What is cumulative cultural evolution? Proceedings of the Royal Society B: Biological Sciences, 285(1880):20180712.

[36] Rendell, L., Boyd, R., Cownden, D., Enquist, M., Eriksson, K., Feldman, M. W., Fogarty, L., Ghirlanda, S., Lillicrap, T., and Laland, K. N. (2010). Why copy others? insights from the social learning strategies tournament. Science, 328(5975):208-213.

[37] Shafto, P., Goodman, N. D., and Frank, M. C. (2012). Learning from others: The consequences of psychological reasoning for human learning. Perspectives on Psychological Science, 7(4):341-351.

[38] Shafto, P., Goodman, N. D., and Griffiths, T. L. (2014). A rational account of pedagogical reasoning: Teaching by, and learning from, examples. Cognitive psychology, 71:55-89.

[39] Sterelny, K. (2012). The evolved apprentice. MIT press.

[40] Thornton, A. and McAuliffe, K. (2006). Teaching in wild meerkats. Science, 313(5784):227-229.

[41] Thornton, A. and Raihani, N. J. (2008). The evolution of teaching. Animal behaviour, 75(6):1823-1836.

[42] van Lawick-Goodall, J. (1968). The behaviour of free-living chimpanzees in the gombe stream reserve. Animal behaviour monographs, 1:161-IN12.

[43] Zwirner, E. and Thornton, A. (2015). Cognitive requirements of cumulative culture: teaching is useful but not essential. Scientific reports, $5(1): 1-8$. 